\title{
Bone resorbing activity in synovial fluids in destructive osteoarthritis and rheumatoid arthritis
}

\author{
W H ALWAN, ${ }^{1}$ P A DIEPPE, ${ }^{2}$ C J ELSON, ${ }^{1}$ AND J W B BRADFIELD ${ }^{1}$ \\ From the Departments of ${ }^{1}$ Pathology and ${ }^{2}$ Medicine, Bristol University, Bristol
}

SUMMARY The synovial fluids of patients with a destructive form of osteoarthritis (DOA) were $\frac{\mathrm{D}^{\circ}}{0}$ shown to contain high levels of bone resorbing activity as judged by the ability of the fluid to stimulate the release of ${ }^{45} \mathrm{Ca}$ from labelled cultured mouse calvariae. The activity was lost on: extended storage of the synovial fluids and was dependent for its effect on cellular activity in $\vec{\oplus}$ bone. Bone resorbing activity was present in most synovial fluids from patients with DOA ando rheumatoid arthritis (RA) but occurred at higher levels in the former. In contrast, interleukin $1_{-}^{\text {? }}$ (IL1) activity, measured by the mouse thymocytes costimulation assay, was higher in RA than 3 DOA synovial fluids. Little or no bone resorbing or IL1 activity was detected in synovial fluids $\stackrel{\frac{N}{\circ}}{\sim}$ from patients with pseudogout or non-destructive osteoarthritis. These results suggest that most ${ }^{-}$ DOA synovial fluids contain a bone resorbing factor other than IL1. It is considered that the $\vec{\infty}_{\infty}$ factor may be produced by synovial cells stimulated by hydroxyapatite crystals.

Key word: interleukin 1 .

Osteoarthritis is a common disease characterised by destruction of articular cartilage with increased activity and remodelling of subchondral bone. A few patients have the disease in a more destructive form so that, in addition to extensive cartilage loss, there is radiological evidence of bone destruction. Clinical features in these patients may include pain, large cool effusions, and restriction of joint movement, with rapid progression of the destructive process. The synovial fluids of such patients are sterile and free from inflammatory cells, but they contain large amounts of hydroxyapatite crystals. Various names have been used to describe this destructive form of osteoarthritis (DOA), including apatite associated destructive arthropathy, ${ }^{1}$ Milwaukee shoulder syndrome, ${ }^{23}$ basic calcium phosphate crystal deposition disease ${ }^{45}$ and analgesic arthropathy. ${ }^{67}$

The mechanisms of joint destruction in osteoarthritis are unknown, though some potential mediators have been investigated. Halverson and colleagues reported that some synovial fluids from patients with DOA contain high levels of activated collagenase and proteases ${ }^{3}$ and that these enzymes are released from cultured synoviocytes stimulated

Accepted for publication 2 September 1987.

Correspondence to Professor J W B Bradfield, Pathology Department, University of Bristol, Bristol BS8 1TD

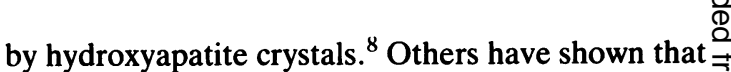
when cultured synovium engulfs hydroxyapatite $\frac{3}{3}$ crystals there is release of prostaglandin $\mathrm{E}_{2},{ }^{9} \mathrm{a}=$ potent bone resorbing agent. If these reactions occur in the patients' joints then they appear to be independent of cellular inflammation, as assessed by histological examination of the patients' synovialo membranes. ${ }^{10}$

Recently a number of bone and cartilage $\delta$ resorbing agents have been described, at least one of $₹$ which, IL $1,{ }^{11-13}$ has been detected in the synovialo fluids from patients with inflammatory arthritides, especially $\mathrm{RA}^{1415}$ and gout. ${ }^{16}{ }^{17}$ IL1 is known to을. stimulate synovial cells to release destructive enzymes in vitro. ${ }^{18}{ }^{19}$ These findings prompted us to 9 examine synovial fluids from patients with DOA for ${ }_{0}^{N}$ bone resorbing and IL1 activity.

\section{Patients and methods}

\section{PATIENTS}

Details of the patients are shown in Table 1.

Patients studied were those attending rheuma- $-\overline{0}$ tology outpatient clinics at the Bristol Royal市 Infirmary and requiring aspiration of synovial fluid $\stackrel{?}{\mathbb{D}}$ from a knee or shoulder for diagnostic or thera- $\frac{\varrho}{Q}$ peutic purposes. A diagnosis of rheumatoid arthritis was made according to the classical American 
Table 1 Source of synovial fluid specimens

\begin{tabular}{ccccc}
\hline \multicolumn{5}{c}{ Type of arthritis } \\
\cline { 2 - 5 } & $D O A$ & $O A$ & $R A$ & $P A^{*}$ \\
\hline $\begin{array}{c}\text { Number of } \\
\text { patients }\end{array}$ & 25 & 10 & 15 & 11 \\
$\begin{array}{c}\text { Sex (M:F) } \\
\text { Age, mean } \\
\text { (range) }\end{array}$ & $5: 20$ & $4: 6$ & $5: 10$ & $7: 4$ \\
\hline
\end{tabular}

*PA=pyrophosphate arthropathy.

Rheumatism Association criteria ${ }^{20}$ or of osteoarthritis (OA) by the presence of typical clinical and radiological features. Those with OA were further subdivided into three groups as follows on the basis of synovial fluid and radiological findings: (a) Destructive or apatite associated OA. These patients had radiological evidence of bone destruction and abundant particles staining with alizarin red in the synovial fluid. The clinical and radiological features of this group have been described previously. ${ }^{1}$ (b) Simple OA. These patients had no radiological evidence of bone destruction or chondrocalcinosis and no crystals in their synovial fluid, but did have painful joints with radiological evidence of joint space narrowing and subchondral bone reaction. (c) Pyrophosphate arthropathy (PA). These patients had radiological evidence of chondrocalcinosis, synovial fluid pyrophosphate crystals, a hypertrophic bone response, and no destruction of bone. ${ }^{21}$
SYNOVIAL FLUIDS

All fluids were tested immediately for the presence of inflammatory cells, and of crystals as shown by alizarin red staining and polarised light microscopy. The fluids were then centrifuged and the supernatants assayed for bone resorbing and IL1 activity.

BONE RESORPTION ASSAY

Bone resorbing activity was assessed quantitatively using the mouse calvarial system. ${ }^{22}$ Briefly, the skeletons of 1-2 day old mice were labelled with ${ }^{45} \mathrm{Ca}$ by subcutaneous injection of $37 \mathrm{kBq}$ of ${ }^{45} \mathrm{CaCl}_{2}$ (Amersham International) in each mouse. Four days later half calvariae were removed and cultured on stainless steel grids in $1.5 \mathrm{ml}$ modified Bigger's medium (Imperial Laboratories), supplemented with $5 \%$ heat inactivated rabbit serum (Wellcome). After 24 hours preincubation bones were transferred either to control medium or to medium containing synovial fluid and cultured for a further 48 hours at $37^{\circ} \mathrm{C}$. Aliquots $(0.5 \mathrm{ml})$ of medium were removed and their radioactivity measured by scintillation counting. The results were expressed as the ratio of ${ }^{45} \mathrm{Ca}$ released into the medium from treated bones to that from control bones; a ratio greater than one indicates the presence of resorptive activity in the synovial fluid. For each experiment positive and negative controls were employed. For positive controls, either vitamin A $22 \mathrm{IU} / \mathrm{ml}^{23}$ (Sigma) or prostaglandin $E_{2} 10^{-6} \mathrm{~mol} / \mathrm{l}^{24}$ (Sigma) was used to stimulate bone resorption. For negative controls, dead calvariae were used. These were prepared by exposing calvariae to three cycles of freezing and

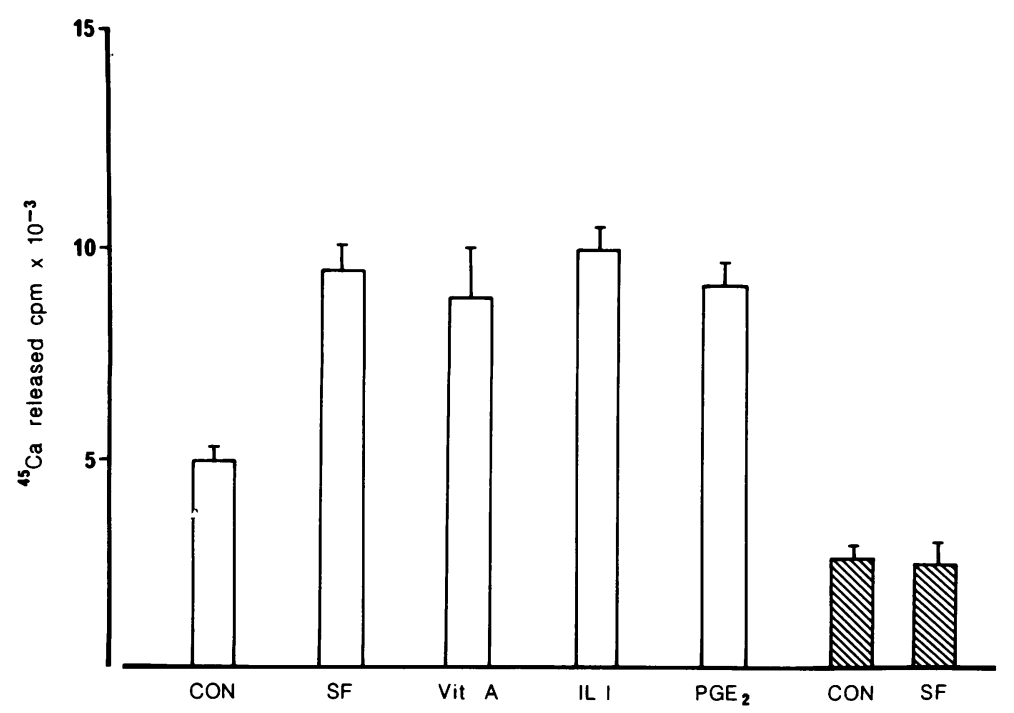

Fig. 1 Effect of $5 \%$ SF from patients with $D O A, 22 I U / \mathrm{ml}$ vitamin $A$ (Vit A), $2 \mathrm{U} / \mathrm{ml} \mathrm{ILI,} \mathrm{and} 10^{-6} \mathrm{M}$ $P G E_{2}$ on calcium release by live mouse calvariae compared with the effect of medium alone (CON). The effect of SF on dead calvariae is also shown. Each experiment was done in triplicate; each result represents the mean of six experiments. Limits represent $1 S D$. The effect is expressed as the amount of ${ }^{45} \mathrm{Ca}$ released into the medium. Shaded columns represent release of calcium from dead control and $S F$ treated bones. 
thawing. At the end of the experiment some treated bones and their controls were fixed in formalin, decalcified, and processed for histological study. Sections were stained with haematoxylin and eosin and examined by light microscopy.

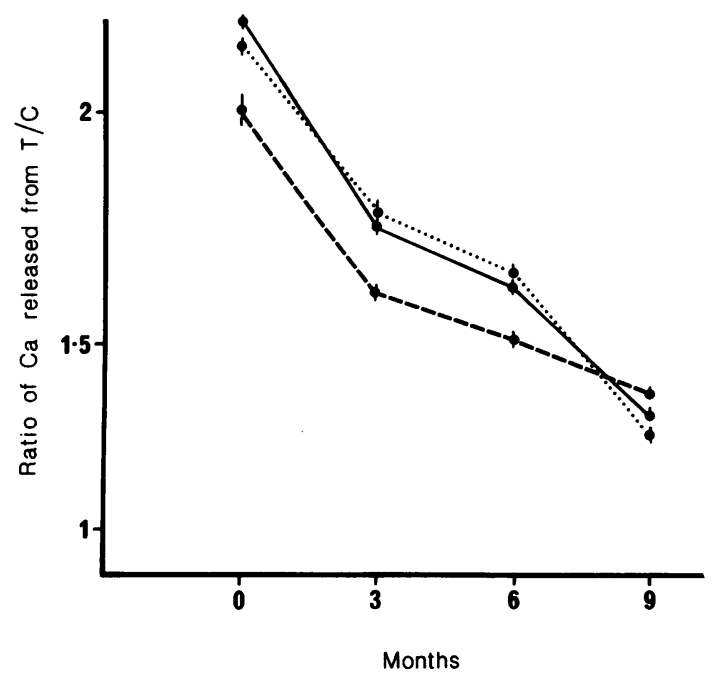

Fig. 2 Effect of storage on bone resorbing activity of SF. Three SF samples were tested at a concentration of $3 \%$ immediately after aspiration and then after storage at $-70^{\circ} \mathrm{C}$ at the intervals shown. The effects are expressed as the mean (SD) of triplicates of each sample and represent the ratio of ${ }^{45} \mathrm{Ca}$ released from treated bone to that released from bone in medium alone $(T / C)$.
IN TERLEU KIN 1 AS SAY

This was performed as described elsewhere ${ }^{25}$ witt? some modifications. Quadruplicate cultures were prepared in microtest well plates (Nunc); eacle contained $2 \times 10^{5}$ thymocytes from $\mathrm{C}_{3} \mathrm{H} / \mathrm{HeJ}$ mice in $200 \mu \mathrm{l}$ of RPMI 1640 medium containing $1 \%$ phytohaemagglutinin (PHA, Gibco) and dilutions of synovial fluid (SF). The SF samples were treate with sheep testis hyaluronidase $100 \mathrm{U} / \mathrm{ml}$ (Sigmaळ before testing. Control wells contained thymocytes alone or thymocytes and PHA. As a positives control, thymocytes were cultured in mediuna containing $0.25 \mathrm{U} / \mathrm{ml}$ purified IL1 (Genzyme). Afte $\bar{p}$ incubation for 48 hours at $37^{\circ} \mathrm{C}$ the thymocytes were pulsed with $37 \mathrm{kBq} / \mathrm{well}$ of $\left[{ }^{3} \mathrm{H}\right]$ thymidine (Amersham) for the last six hours and harvested with an automatic cell harvester. Thymidine uptake was measured in a liquid scintillation counter. Datæ were expressed as the mean (SD) of quadruplicate $\overrightarrow{\mathrm{e}}$ cultures.

STATISTICAL ANALYSIS

Student's $t$ test was used to compare the control an experimental groups in both assays.

\section{Results}

BONE RESORBING ACTIVITY OF DOA SYNOVIAL FLUID

Fig. 1 shows that labelled calvariae cultured with DOA synovial fluid released more radioactivity tha calvariae cultured in medium alone $(p<0.001)$. The known bone resorbing agents, vitamin $\mathrm{A}, \mathrm{IL1}$, and

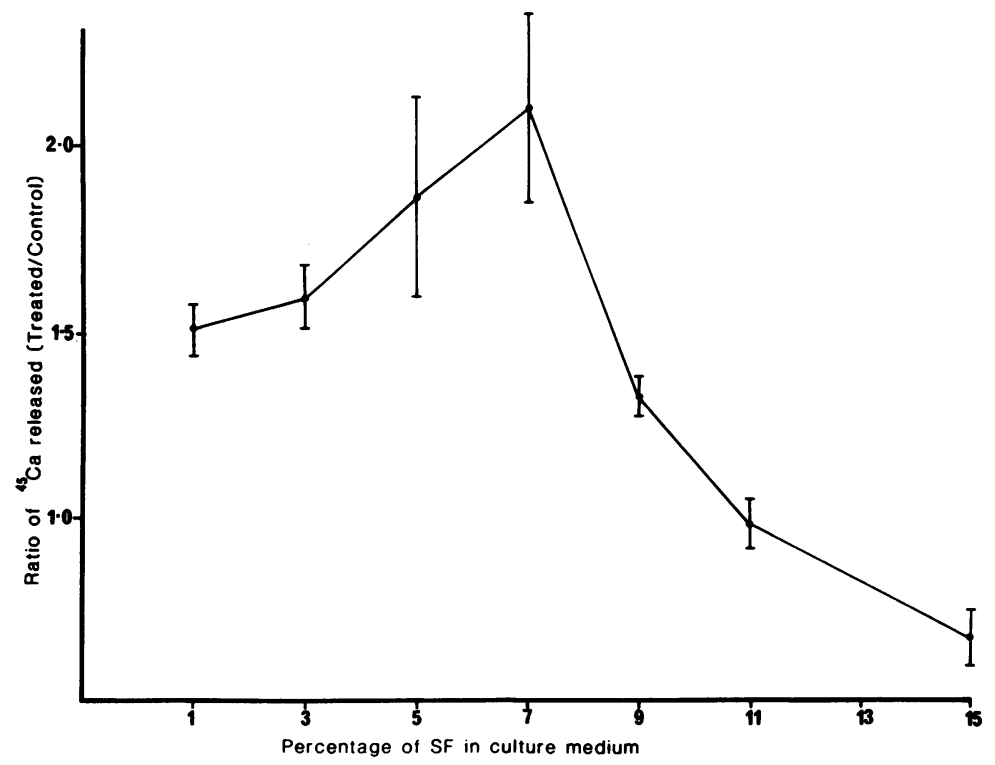

Fig. 3 Relation between concentration and bone resorbing activity of synovial fluid from 24 patients with DOA. The results are expressed as the ratio of ${ }^{45} \mathrm{Ca}$ release from treated bones to that released from controls and are the mean (SD) 
$\mathrm{PGE}_{2}$, induced a similar regponse. In contrast, SF failed to stimulate the release of calcium from dead bones.

It was noticed that SF appeared to have reduced bone resorbing activity on storage. To verify this observation samples were stored at $-70^{\circ} \mathrm{C}$ and repeatedly thawed for each testing at intervals. Fig. 2 shows the result of experiment with three fluids. In each case there was a progressive loss of activity with time. In a further experiment (not illustrated) aliquots of the initial sample were obtained at the time of initial storage and only one sample thawed at each time. Activity again diminished with time, which suggests that the loss of activity is associated with length of time of storage rather than with the effect of repeated thawing and refreezing. Because of this all subsequent experiments on bone resorbing activity were conducted on fresh synovial fluids.

Experiments were set up to test the effect of varying the concentration of synovial fluid on bone resorbing activity. Synovial fluids with bone resorbing activity from patients with DOA were diluted and added to live labelled bones. Fig. 3 shows that there was stimulation of bone resorption at the lowest concentration tested, which was $1 \%$. Peak resorption occurred at concentrations between $5 \%$ and $7 \%$. At levels above $10 \%$ no further enhancement of calcium release was found; on the contrary, high concentrations inhibited bone resorption.

To test if the reduced effect at high concentrations of synovial fluid was due to a toxic effect on the cultured bone, high concentrations of SF were added to cultures stimulated with vitamin $\mathrm{A}$. Whereas the ratio of ${ }^{45} \mathrm{Ca}$ released from vitamin $\mathrm{A}$ treated bones to that released from control bones was $1 \cdot 6$, the addition of $20 \%$ SF from patients with DOA reduced the ratio to less than 1 (complete inhibition). Suboptimal concentrations of SF $(1-2 \%)$ had no effect on vitamin $\mathrm{A}$ induced ${ }^{45} \mathrm{Ca}$ release.

Fig. 4 shows a comparison between the levels of bone resorbing activity in DOA synovial fluids and those in SF from other arthritides. High levels of activity were present in 24/25 of DOA synovial fluids, whereas little or no activity was detected in fluids from patients with OA and PA. Most (12/15) RA fluids were active, but the average level was significantly less than that found in DOA $(\mathrm{p}<0 \cdot 05)$. This could not be attributed to variations in the assay as the amount of ${ }^{45} \mathrm{Ca}$ release induced by vitamin $\mathrm{A}$, the positive control in these experiments, fell within the range shown for vitamin A in Fig. 1. The possibility that this difference resulted from the RA fluids being tested at a suboptimal concen-

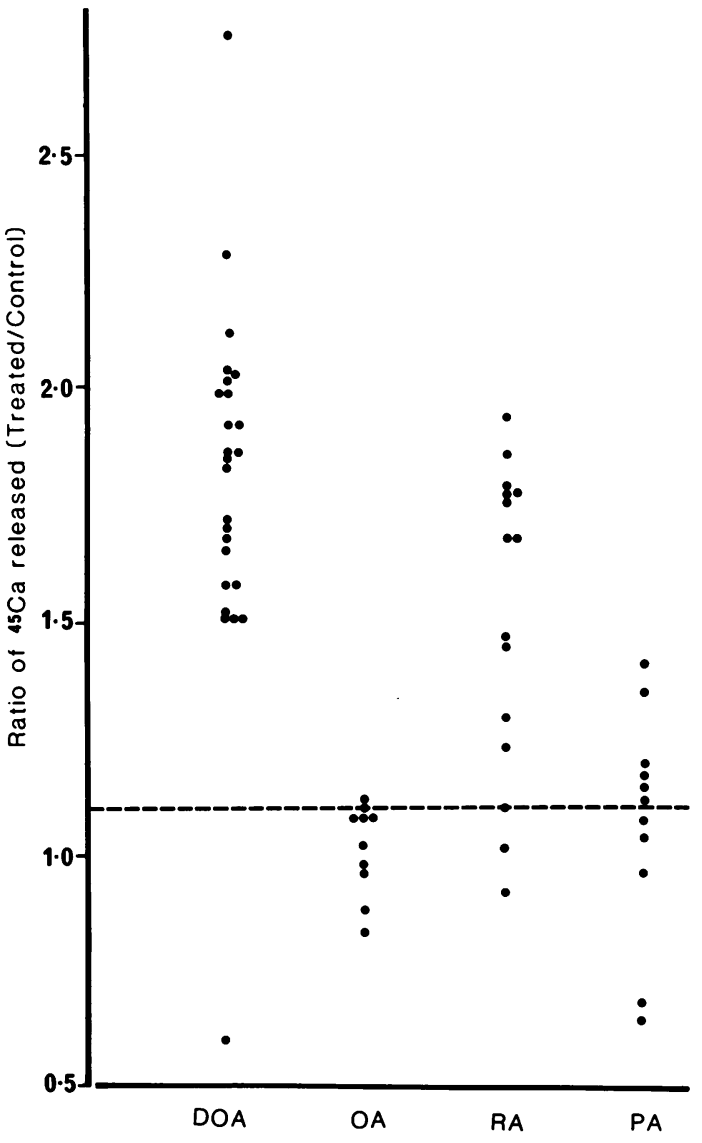

Fig. 4 Comparison of bone resorbing activity in $S F$ from different arthritides. The fluids were tested at a final concentration of $5 \%$. The activity is expressed as the ratio of ${ }^{45} \mathrm{Ca}$ released from treated bone to that from bone in medium alone. The dotted line represents the upper limit of calcium release which is usually accepted as non-significant.

tration was investigated by employing different concentrations of RA fluids. The results showed that, as with DOA, maximal activity occurred at a concentration of $6 \%$. The three RA synovial fluids which were inactive in the initial test (Fig. 4) showed no activity at any of the dilutions tested.

\section{HISTOLOGICAL EXAMINATION}

The results recorded in Fig. 1 show that calcium release from bone induced by SF depends on live cells in the bone, presumably osteoclasts. To investigate this, histological sections were prepared from living bones which had been incubated with either synovial fluid or with medium alone. Although there appeared to be more osteoclasts in 
the former group (Fig. 5b) than in the latter (Fig. 5a), multinucleated osteoclasts were seen in the latter group. Thus such histological observations should be treated with some caution.

INTERLEUKIN 1 ACTIVITY OF DOA AND

OTHER SYNOVIAL. FLUIDS

The effect of different concentrations of DOA and RA synovial fluids on thymocyte proliferation was measured. Fig. 6 shows that fluids from both groups of patients gave maximum proliferation at a concen- tration of $3 \%$. Although the curves for both groupse were similar, the fluids from patients with DOA induced less proliferation than those from patients? with RA.

Fig. 7 compares the thymocytes co-mitogenic stimulating activity of fluids from patients with DOA, OA, RA, and PA, all tested at a concen- $\triangle$ tration of $3 \%$. The results show that synovial fluids from patients with $\mathrm{OA}$ and PA lacked activity, whereas fluids from most patients with RA and some with DOA stimulated thymocyte prolifera $\overrightarrow{\bar{\omega}}$

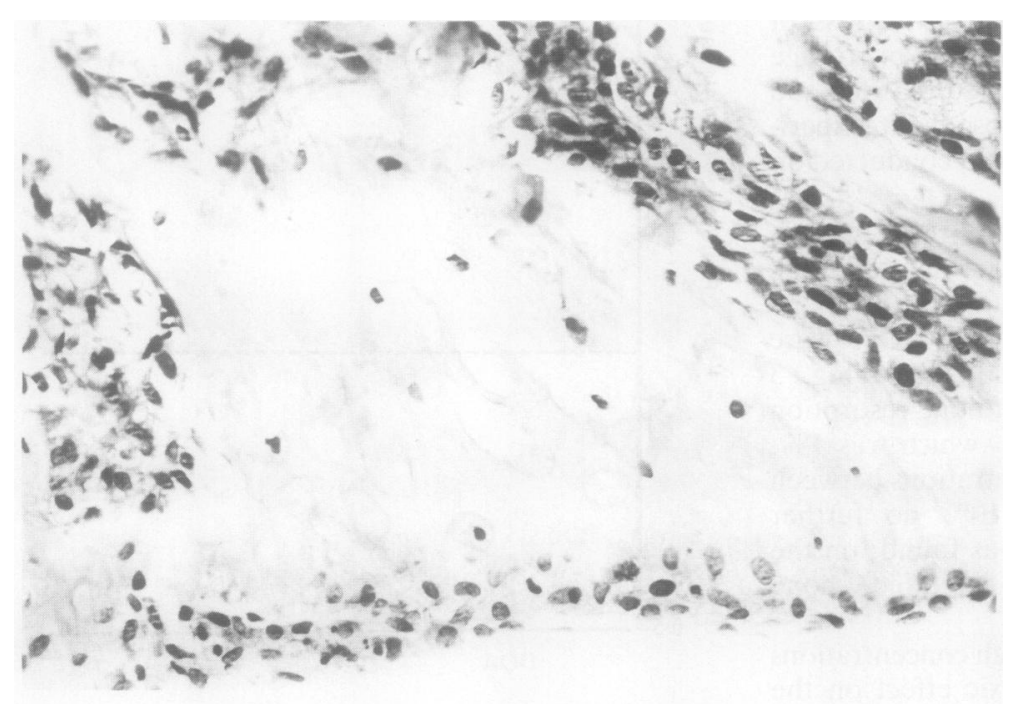

(a)

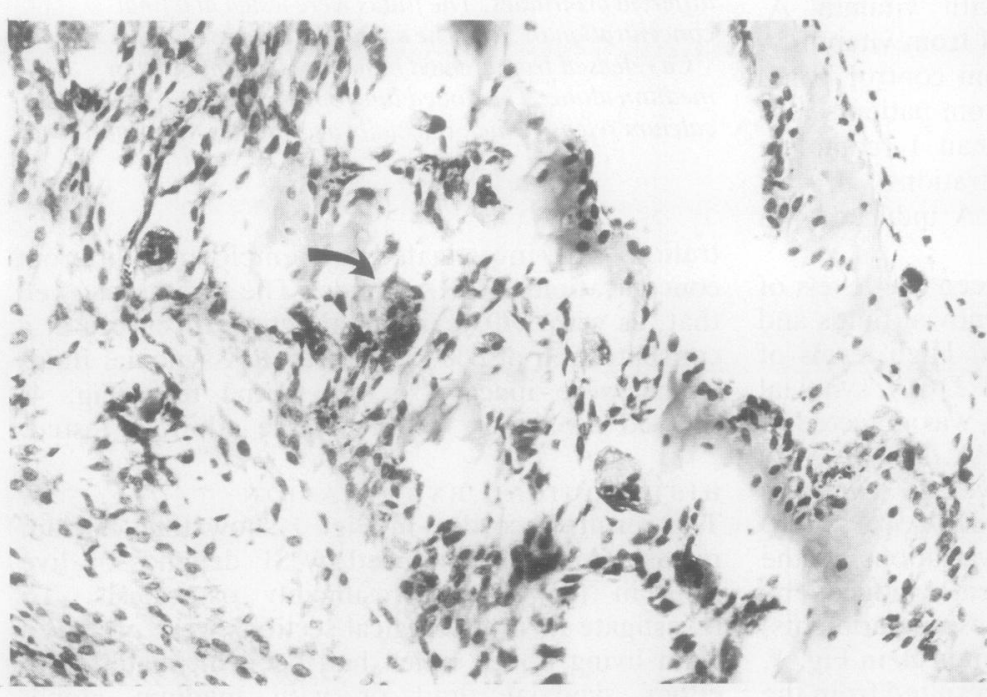

(b)
Fig. 5 Photomicrographs of calvariae. (Haematoxylin and eosin.) (a) In the control tissue there is solid bone with surrounding mononucleated cells with few if any osteoclasts. (b) In a typical area from tissue treated with SF the bone shows multiple lacunae associated with numerous multinucleated giant cells (arrow). 


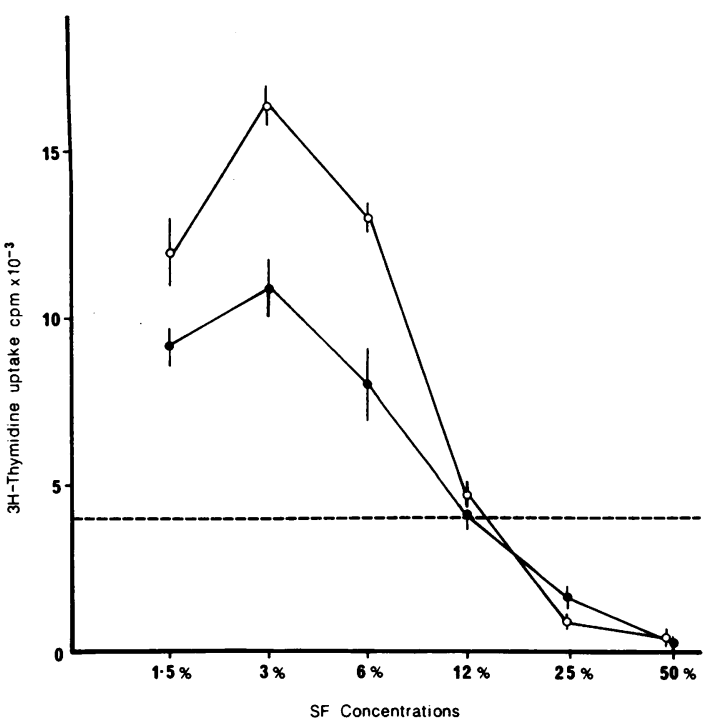

Fig. 6 Thymocytes co-mitogenic stimulating ILI activity in $S F$ from 13 patients with $R A(O)$ and six patients with $D O A(O)$ at different concentrations. The results are expressed as the mean $(S D)$ of $\left[{ }^{3} H\right]$ thymidine uptake by thymocytes after 48 hours' culture at each concentration. The dotted line represents the upper limit of activity given by PHA alone.

tion. As a group rheumatoid fluids were significantly more active than those from patients with DOA $(\mathrm{p}<0 \cdot 001)$.

\section{DIALYSIS OF DOA SYNOVIAL FLUIDS}

Preliminary experiments were set up to gain some insight into the chemical nature of the bone resorbing agent in DOA synovial fluids. DOA synovial fluids were tested before and after dialysis against medium for 48 hours at $4^{\circ} \mathrm{C}$. The results showed that all synovial fluids had reduced bone resorbing activity after dialysis. In some (1-4) the activity was lost altogether, whereas in others $(5$ and 6) some activity still remained (Table 2 ). The latter had detectable levels of IL1 activity (greater than $10000 \mathrm{cpm}$, see Fig. 7), whereas the former had insignificant levels $(6000 \mathrm{cpm}$ or less).

\section{Discussion}

The main conclusion from this study is that synovial fluid from patients with the rapidly destructive form of osteoarthritis contains one or more bone resorbing agents. The activity of the fluids was comparable in magnitude with that of other known bone resorbing agents such as vitamin A, IL1, and

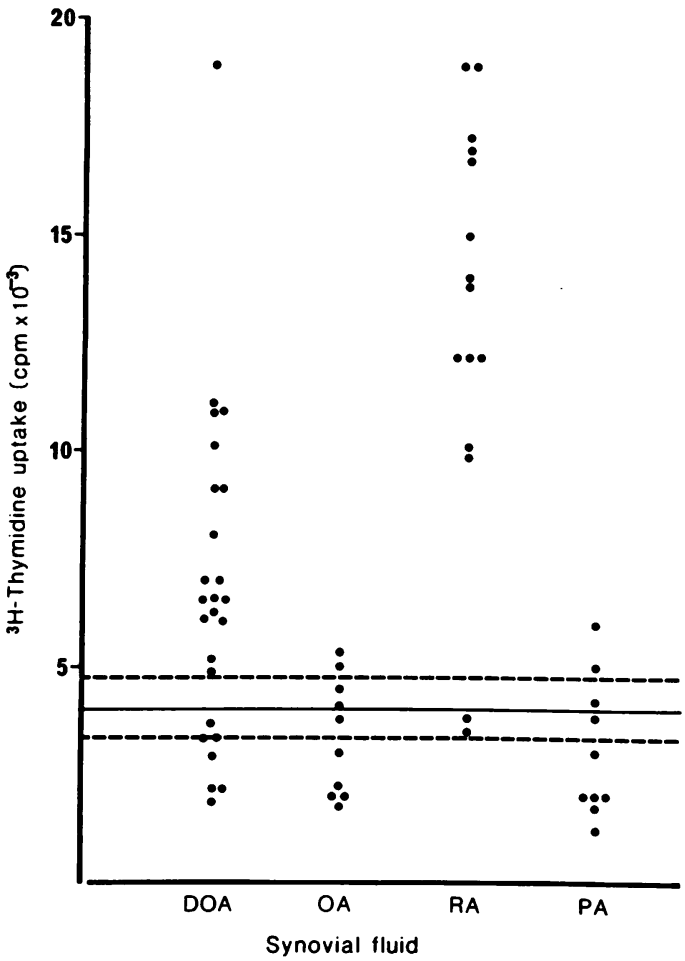

Fig. 7 Comparison of ILI activity in SF from different arthritides. The fluids were tested at a final concentration of $3 \%$. The results are expressed as the tritiated thymidine uptake by thymocytes after 48 hours' culture. The dotted lines define ISD on either side of the mean given by PHA alone.

Table 2 Effect of dialysis on bone resorbing activity of synovial fluid

\begin{tabular}{|c|c|c|}
\hline$S F^{*}$ & $T / C$ ratio before dialysis $t$ & $T / C$ ratio after dialysis \\
\hline 1 & $1.58(0.03) \ddagger$ & $0.98(0.08)$ \\
\hline 2 & $1.29(0.01)$ & $0.91(0.10)$ Little or \\
\hline 3 & $1.69(0.04)$ & $1.07(0.08)$ \\
\hline 4 & $1.79(0.07)$ & $0.97(0 \cdot 10)$ \\
\hline 5 & $1.81(0.03)$ & $1.39(0.06)$ Some \\
\hline 6 & $1.76(0.01)$ & $1.31(0.05) j$ \\
\hline
\end{tabular}

${ }^{*}$ Fluids were tested at a concentration of $3 \%$, and six bones were used for each synovial fluid.

$\dagger \mathrm{T} / \mathrm{C}=$ treated/control.

$\ddagger$ Results are mean (SEM).

$\mathrm{PGE}_{2}$. Moreover, their mechanism of action appears similar in some respects. As the fluids failed to cause release of calcium from dead bones their mode of action cannot be due to direct dissolution of hydroxyapatite crystals but must be due to the stimulation of cells in the bone, which then modified 
resorption. A similar dependence on the presence of healthy live osteoclasts (and osteoblasts) has been reported for bone resorption induced by parathormone, ${ }^{22}$ osteoclast activating factor, ${ }^{26}$ and IL1. ${ }^{13}$ Further evidence of osteoclast activation was sought in this study by examining the histological appearances of bone incubated with SF and comparing these with the appearances of bone which had been incubated with medium alone. Although increased numbers of large multinucleated osteoclasts seemed to be present in the former group, similar, though fewer. cells were present in the control tissues. Confidence in these findings would require a full morphometric analysis of large numbers of sections.

The bone resorbing activity of SF was most marked at concentrations between $5 \%$ and $7 \%$. At higher concentrations the effect was less marked. This could be due to high concentrations of the bone resorbing agent(s) down regulating its own activity, to a specific inhibitor of the agent coming in to play as its concentration increases, or to high concentrations of synovial fluid providing an unfavourable environment for cellular activity in the bone. The fact that high concentrations of DOA synovial fluids prevented vitamin A from exerting its bone resorbing effect provides evidence in support of the last hypothesis.

It was considered that the bone resorbing activity in synovial fluids from patients with DOA might be due to IL1. When these fluids were assayed for IL1 activity using a standard mouse thymocyte comitogenic assay, ILl-like activity was indeed found in some of the fluids, but other fluids which stimulated bone resorption failed to show ILl-like activity. In addition, when fluids from patients with DOA and RA were compared there was a discrepancy between the two types of assay. Fluids from the patients with DOA stimulated more bone resorption than those from patients with RA, whereas the reverse was true for IL1-like activity. Our interpretation of these results is that although ILl may contribute to the bone resorbing activity in both DOA and RA fluids, a different factor, possibly $\mathrm{PGE}_{2},{ }^{9}$ is responsible for most of the bone resorbing activity in DOA. This possibility gains credence from the finding that much of the activity in DOA synovial fluids was dialysable. Indeed, even in RA synovial fluid other bone resorbing factors are likely to be present as recent work has shown that tumour necrosis factor has bone resorbing activity $^{27}$ and high levels of tumour necrosis factor messenger RNA have been extracted from RA synovial cells (Buchan $\mathrm{G}$, personal communication).

The destructive form of osteoarthritis is characterised by the presence of hydroxyapatite crystals in the synovial fluid and degeneration of the articular $\frac{0}{0 .}$ cartilage with exposure of the underlying bone and $\Rightarrow$ extensive destruction and resorption of the latter. We have preliminary evidence that hydroxyapatite crystals can stimulate macrophages to release a $\frac{\bar{D}}{\bar{D}}$ factor which shows bone resorbing activity but not $\frac{\text { के }}{7}$ IL1-like activity. At present it is unclear to what $\stackrel{\mathbb{Q}}{\varrho}$ extent the production of such bone resorbing factors can explain the relation between hydroxyapatite $\vec{\circ}$ crystals and bone resorption in DOA. Current work is directed towards characterising the bone $\vec{\omega}$ resorbing factor in order to compare it with other known resorbing agents.

This work was supported in part by grants from the Arthritis and Rheumatism Council and MRC. Dr Alwan was in receipt of an overseas research student award. We would like to thank Dr Papoian for his help and advice

\section{References}

1 Dieppe P'A. Doherty M. MacFarlane D G. Hutton C' W. Bradficld J W. Watt I. Apatite associated destructive arthritis. Br J Rheumatol 1984; 23: 84-91.

2 McCarty D J, Halverson P B, Carrera G F, Brewer B J. Kozin F. 'Milwaukee shoulder'-association of microspheroids containing hydroxyapatite crystals. active collagenase, and neutral protease with rotator cuff defects. I. Clinical apsects. Arthritis Rheum 1981: 24: 464-73.

3 Halverson P B, Cheung IIS. McCarty D J. Garancis J. Mandel N. 'Milwaukee shoulder-association of microspheroids containing hydroxyapatite crystals, active collagenase, and neutral $\mathbb{D}$ protease with rotator cuff defects. II. Synovial fluid studies. Arthritis Rheum 1981: 24: 474-83.

4 Halverson P B. McCarty D J, Cheung H S. Ryan L M. Milwaukec shoulder syndrome: eleven additional cases with $\supsetneq$ involvement of the knee in seven (basic calcium phosphate crystal deposition diseasc). Semin Arthritis Rheum 1984: 14: 36-44.

5 Halverson P B. Garancis J C. McCarty D J. Histopathological and ultrastructural studies of synovium in Milwaukec shoulder syndrome-a basic calcium phosphate crystal arthropathy. Ann Rheum Dis 1984; 43: 734-41.

6 Ronningen H. Langeland $N$. Indomethacin treatment in 3 OA of the hip joint. Acta Orthop Scand 1979: 50: 169--74.

7 Doherty M. Holt M. MacMillan P, Watt I, Dieppe P. 옥 A reappraisal of 'analgesic hip'. Ann Rheum Dis 1986; 45: I 272-6.

8 Cheung H S. Halverson P B, McCarty D J. Release of collagenase, neutral protease and prostaglandins from cultured $\mathcal{N}$ mammalian synovial cells by hydroxyapatite and calcium pyrophosphate crystals. Arthritis Rheum 1981; 24: 1338-44.

9 McCarty D J, Cheung H S. Prostaglandin (PG)E, generation N by cultured canine synovial fibroblasts exposed to microcrystals containing calcium. Ann Rheum Dis 1985; 44: 316-20.

10 Alwan W H, Dicppe P A. Watt I, Bradficld J W B. Pathologye of apatite associated destructive arthropathy [Abstract]. J Pathol 1985; 146: 254A.

11 Gowen M, Wood D D. Ihrie E J, McGuire M K B, Russcll R G G. An interleukin 1 like factor stimulates bone resorption in vitro. Nature 1983; 306: 378-80.

12 Gowen M, Wood D D, Ihric E J, Mcats J E, Russell R G G. D Stimulation by human interleukin 1 of cartilage breakdown and $\vec{D}$ production of collagenase and proteoglycanase by human chondrocytes but not by human osteoblasts in vitro. Biochim Biophys Acta 1984: 797: 186-93 
13 Heath J K, Saklatvala J, Meikle M C, Atkinson S J, Reynolds J J. Pig interleukin 1 (catabolin) is a potent stimulator of bone resorption in vitro. Calcif Tissue Int 1985; 37: 95-7.

14 Bendtzen K, Peterson J, Halkjaer-Kristensen J, IngemannHansen T. Interleukin 1-like activities in synovial fluids of patients with rheumatoid arthritis and traumatic arthritis. Rheumatol Int 1985; 5: 79-82.

15 Fontana A, Hengartner H, Weber E, Fehr K, Grob P J, Cohen G. Interleukin 1 activity in synovial fluid of patients with rheumatoid arthritis. Rheumatol Int 1982; 2: 49-53.

16 Malawista S E, Duff G W, Atkins E, Cheung H S, McCarty D J. Crystal-induced endogenous pyrogen production. A further look at gouty inflammation. Arthritis Rheum 1985; 28: 1039-46.

17 Woolf A D, Nouri A M E, Woo P, Richter M B, Panayi G S, Gibson $\mathrm{T}$. Interleukin 1 and the acute phase response in acute crystal synovitis. Br J Rheumatol 1985; 24 (suppl 1): 203-8.

18 Dayer J M, Rochemonteix B, Burrus B, Demczuk S, Dinarello C A. Human recombinant interleukin 1 stimulates collagenase and prostaglandin $E_{2}$ production by human synovial cells. $J$ Clin Invest 1986; 77: 645-8.

19 Richardson H J, Elford P R, Sharrard R M, Meats J E, Russell R G G. Modulation of connective tissue metabolism by partially purified human interleukin 1. Cell Immunol 1985; 90: $41-51$.
20 Ropes M W. Bennett G A, Cobb S. Jacox R. Jessar R A. Revision of diagnostic criteria for rheumatoid arthritis. Bull Rheum Dis 1958; 9: 175-6.

21 Dieppe P A. Watt I. Crystal deposition in osteoarthritis: An opportunistic event? Clin Rheum Dis 1985; 11: 367-92.

22 Reynolds J J. Organ cultures of bone: studies on the physiology and pathology of resorption. In: Balls M. Monnichendam M. eds. Organ culture in biomedical research. Cambridge: Cambridge University Press, 1976: 355-66.

23 Barnicot N A. The local action of vitamin A on bone. J Anat 1950; 84: 374-87.

24 Klein D C, Raisze L G. Prostaglandins: stimulation of bone resorption in tissue culture. Endocrinology 1970; 86: 1436-40.

25 Gery I, Gershon R K. Waksman B H. Potentiation of the T-lymphocytes response to mitogens. J Exp Med 1972; 136: $128-42$.

26 Bockman R S, Repo M A. Lymphokine-mediated bone resorption requires prostaglandin synthesis. J Exp Med 1981; 154: 529-34.

27 Bertolini D R, Nedwin G E, Bringman T S, Smith D D, Mundy G R. Stimulation of bone resorption and inhibition of bone formation in vitro by human tumour necrosis factors. Nature 1986; 319: 516-8. 